\title{
Photothermal characterisation of surface hardened steel
}

\author{
by T.T.N. Lan', D.T. Son ${ }^{2}$ and H.G. Walther' \\ ${ }^{1}$ Institut für Optik und Quantenelektronik, Friedrich-Schiller-Universitat Jena, Max-Wien-Platz 1, D-07743 Jena, \\ Germany; ${ }^{2}$ Institut für Fertigung, Technische Universităt IImenau, Postfach 0565, D-98684, Gemany
}

\begin{abstract}
Photothermal measurements at varied modulation frequencies allow for the estimation of depth profiles of thermal diffusivity or thermal conductivity $k$ in case of known thermal density. We suggest an inversion algorithm performing a sequence of one-parameter fits in order to estimate thermal conductivity $k$ profiles. Surface problems in photothermal depth profiling and an approach to overcome them are discussed. Experimental results of thermal conductivity depth profiling of laser-handened steel, based on this inversion algorithm are presented. The photothermally obtained $\mathbf{k}$-profiles are interpreted and compared with results from conventional destructive measuring techniques.
\end{abstract}

\section{Introduction}

The propagation of thermal waves in solids is strongly influenced by the material's microscopic properties, by its physical microstructure, and especially by the distribution of inner interfaces. Internal interfaces limit the free path length of carriers of inner energy in a solid such as electrons or phonons and therefore reduce the thermal conductivity. The interrelation between microstructure and local thermal properties of thermal conductivity $k$ and thermal density $\rho c$ in solids and the fact that the penetration depth of thermal waves in a periodically illuminated sample can be controlled by the modulation frequency $f$ make it possible to establish a novel nondestructive technique of photothermal depth profiling.

The frequency-dependent and consequently depth-dependent photothermal signals carry information on local thermal properties. To reconstruct their depth profile the measured frequency sweep of the signal proportional to the oscillating surface temperature has to be "inverted ".

Ordinary technical surfaces and metal surfaces under investigation are usually neither clean nor optically flat. So, photothermal amplitude measurements fail to characterize the material because the signal amplitude is proportional to the absorptivity of the surface which is out of interest. We report a possibility to overcome this surface problem in reconstruction by using only photothermal phase data. In this paper the suggested inversion algorithm is applied for the photothermal characterisation of laser-hardened steel to show the possibility of quantitative nondestructive estimation of hardness depth profiles.

\section{Theory}

To interpret photothermal measuring data the oscillating surface temperature of the sample under study has to be calculated. For simplification we consider a surface absorbing sample with a depth profile of thermal conductivity $k$ approximated by a polygonal curve ,i.e., $k$ varies piecewise linearely with depth $z$. The thermal density $\rho c$ is assumed to be constant and known [1]. The heating beam spot has to be broad enough to meet the condition of one-dimensional thermal wave propagation.

The region from the sample surface $z=0$ to the depth $z_{N}$ limiting the domain of the depth dependent $\mathrm{k}$ is subdivided into $\mathrm{N}$ virtual sublayers. The thermal wave propagation in such a sample can be described by the matrix formalism, where the surface temperature oscillation can be expressed by a product of $\mathrm{N}$ component matrixes. Each of these matrixes transforms thermal waves from one sublayer to the next [3]. 
The measured frequency sweep of the photothermal signal $S_{\text {meas }}(f)$ has to be inverted to find such a polygonal curve of the $\mathrm{k}$-depth profile corresponding to the real material microstructure depth profile. The inversion technique described in detail in [1] requires the known bulk value $k_{b}$ of the untreated material. k-values at all virtual interfaces $z=z_{m}$ under the surface have to be found. The k-profile is " built-up " step by step from the surface to the bulk of the sample using measured data from high frequencies to lower ones. The advantage of this approach consists in the possibility to retrieve the unknown depth profiles by performing a sequence of one-parameter fits ( $k_{m}$ acts as the only parameter in each step ) instead of using multiparameter methods.

In a former attempt [1] we estimated the unknown $k$ surface value by the normalized signal amplitude measured at sufficiently high modulation frequency. But for many cases the untreated surface used as a reference does not have the same optical absorptivity and infrared emissivity as the modified sample surface. For this reason the phase data are prefered to amplitude data. The $k$ profile is reconstructed from different, guessed surface values $k_{0}$. The calculated phase data curves $S\left(f, k_{0}\right)$ are " fitted " step by step to the measured $S_{\text {meas }}(f)$ one starting from the highest modulation frequency. We can find out the " true " $k_{0}$ by searching for the minimum standard deviation of $S\left(f, k_{0}\right)$ from $S_{\text {meas }}(f)$.

$$
\left\{\frac{1}{N F-1} \sum_{n=1}^{N F}\left[S\left(f_{n}, k_{0}\right)-S_{\text {meas }}\left(f_{n}\right)\right]^{2}\right\}^{\frac{1}{2}}=\operatorname{Min} !
$$

NF is the number of measured frequencies.

\section{Application}

We investigated laser hardened $\mathrm{C60}$ steel samples to test the suggested inversion technique. Plates made of $\mathrm{C} 60$ steel were hardened by a powerful $\mathrm{CO}_{2}$ laser illuminating along two straight lines at different speeds. The laser absorption induced heating of the material was followed by a cooling process in steel itself causing partial microstructure transformation from austenite to martensite increasing the near surface region hardness. As we know from former investigations on case hardened steel a interrelation between the microstructure and the local thermal conductivity exists [2].

For depth profiling these laser hardened steel samples were photothermally measured by a radiometric technique. The infrared radiation emitted from the periodically heated workpieces was received by a liquid nitrogen cooled broadband IR detector. An Argon ion laser used as the heating source was intensity modulated by an acousto-optic modulator. The modulation frequency covered the range from $1 \mathrm{~Hz}$ to $1000 \mathrm{~Hz}$. The signal to noise ratio at $1 \mathrm{~Hz}$ for the amplitude was better than 200. We compared the frequency dependent signals from hardened and untreated surface regions.

Figure 1 shows two measured photothermal phase difference curves representing the base for the data inversion. Applying the numeric technique sketched above and assuming constant thermal density the thermal conductivity profiles were reconstructed. The laser hardened region could be seen by eyes. There the optical absorptivity was different from that of the untreated region. We have to perform the reconstruction procedure with a variation of the surface value $k_{0}$ and estimate the corresponding polygonal k-curves. For each of them the calculated standard deviations are summerized in figure 2. A clear minimum is found at $k_{0}=0.28 \mathrm{~W} / \mathrm{Kcm}$, pointing out the true surface value. The corresponding k-profiles are shown in figure $3 a$ and the corresponding phase data calculated are depicted in figure 1 by solid lines. The same symbols in all figures denote the same sample.

The nondestructively photothermally estimated $\mathrm{k}$ profiles in figure $3 a$ are now compared with the accompanied microhardness profiles in figure $3 b$ obtained by a series of Vickers micro-intdenter measurements on the cross section of the cut samples. 
Comparison of figs. 3(a) and $3(b)$ demonstrate the close anti-correlation between microstructure controlled hardness and local thermal conductivity. The presented results show the ability of photothermal measurements to reconstruct depth profiles of thermal parameters and, for example, to estimate hardness depth profiles quantitatively in a remote and nondestructive manner.

\section{Acknowledgements}

This work was supported by the Graduate Scholarship Program of the Federal State Thüringen and the German Industry Research Foundation.

\section{REFERENCES}

[1] LAN (T.T.N.), SEIDEL (U.) and WALTHER (H.G.). - Theory of microstructural depth profiling by photothermal measurements. J. Appl.Phys. 77, 1995, p. 4739-4745.

[2 LAN (T.T.N.), SEIDEL (U.), WALTHER (H.G.), GOCH (G.) and SCHMITZ (B.). - Expenimental results of photothermal microstructural depth profiling. J. Appl.Phys. 78, 1995, p. 4108-4111.

[3] LAN (T.T.N.) and WALTHER (H.G.).- Photothermal depth profiling using only phase data, J. Appl.Phys., Nov. 1996.

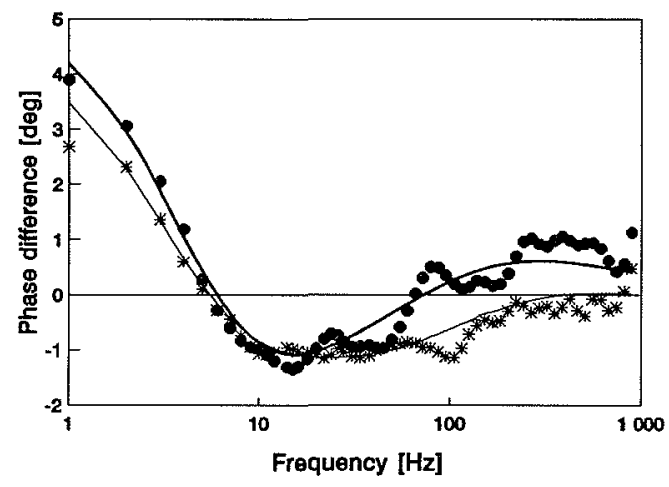

Fig.1. Measured photothermal radiometric phase data for two laser hardened steel samples (symbols) and fitted phase curves (solid lines). 


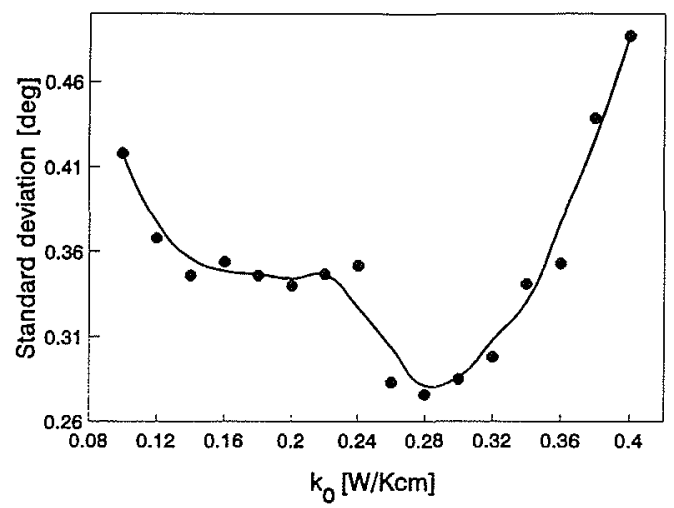

Fig.2. Standard deviation of the calculated phase curve from the measured data curve at various guessed surface values $k_{0}$.

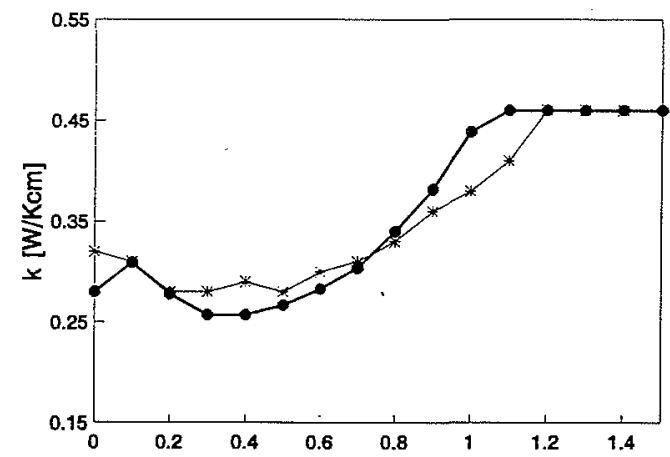

(a)

Depth [mm]

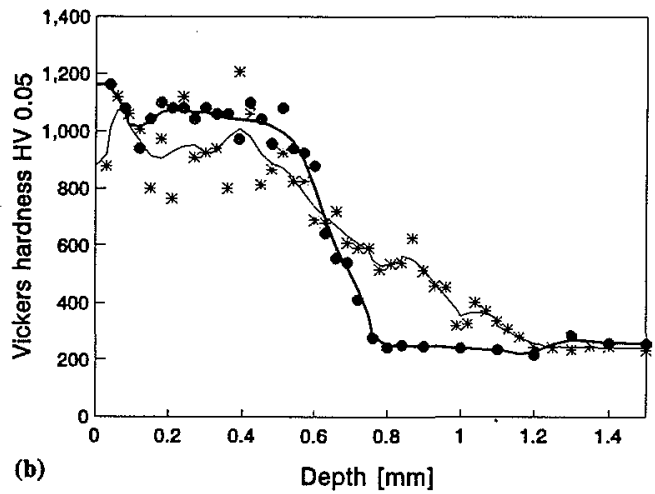

Fig.3. (a). Reconstructed $k$ profiles of two laser hardened steel samples.

(b). Measured Vickers microhardness profiles of the samples in Fig.3.(a). 